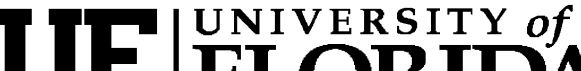 FLORIDA \\ IFAS Extension
}

\section{Weed Control in Beans and Peas (Bush, Pole, Lima Beans, English Peas and Southern Peas) 1}

William M. Stall ${ }^{2}$

Weeds are a major problem in bean and pea production in Florida. Weeds can reduce yields through direct competition for light, moisture and nutrients as well as harbor insects and diseases that attack these crops. Early season competition of weeds is extremely critical and a major emphasis on control should be made during this period. Weeds present at harvest reduce harvest efficiency and increases mechanical damage to the pods.

Some form of bean or pea is planted in every month in some area of Florida. Over this period and area, the variable climatic conditions and soil types influence the diversity of weed species present and their severity. Growers should plan a total weed control program that integrates chemical, mechanical and cultural methods to fit their weed problems and production practices.

Herbicide performance depends on weather, irrigation and soil type as well as proper selection for weed species to be controlled and accurate applications and timing. Obtain consistent results by reading the herbicide label and other information about proper application and timing of each herbicide.

There has been some confusion on certain beans and peas as far as labeling. The southern pea is a Vigna species. Vignas are considered beans. Therefore if the term "beans" appear on the label, the material may be applied to both the Phasealus and Vigna types. These include snap beans, lima beans and southern peas. If a label states "green beans" it may only be applied to green color beans, while the term "snap bean" may also be applied to the wax types. "Peas" do not include the southern pea, but include English peas and Pigeon peas. Make sure you read the label carefully for each commodity that is grown.

To avoid confusion between commercial formulations, suggested rates listed in Table 1 are stated in pounds active ingredient per acre (lbs ai/acre). On marl, rockdale and sandy soils with low organic matter, the lower rates should be applied.

1. This document is HS188, one of a series of the Horticultural Sciences Department, Florida Cooperative Extension Service, Institute of Food and Agricultural Sciences, University of Florida. Original publication date January 1, 2000. Revised October 2, 2006. Visit the EDIS Web Site at http://edis.ifas.ufl.edu.

2. William M. Stall, professor, Horticultural Sciences Department, Cooperative Extension Service, Institute of Food and Agricultural Sciences, University of Florida, Gainesville, 32611.

The use of trade names in this publication is solely for the purpose of providing specific information. It is not a guarantee or warranty of the product named, and does not signify that they are approved to the exclusion of others of suitable composition.7.1.1 
When applying a herbicide for the first time in a new area, use in a small trial basis first.

Before application of a herbicide, carefully read and follow the label. 
Table 1. Chemical weed controls: Beans and Peas.

\begin{tabular}{|c|c|c|c|c|}
\hline \multirow[t]{2}{*}{ Herbicide } & \multirow[t]{2}{*}{ Labeled crops } & \multirow{2}{*}{$\begin{array}{c}\text { Time of } \\
\text { application to crop }\end{array}$} & \multicolumn{2}{|c|}{ Rate (Ibs. Al./Acre) } \\
\hline & & & Mineral & Muck \\
\hline Bentazon (Basagran) & Beans, Peas & Early postemergence & $0.5-1.0$ & $-\cdots$ \\
\hline \multicolumn{5}{|c|}{$\begin{array}{l}\text { Remarks: Apply early postemergence when weeds are small and actively growing. Beans are to Basagran after the } \\
\text { first trifoliate leaf has fully expanded. A crop oil concentrate or a UAN solution (28,30,32\% nitrogen solution) may be } \\
\text { added for improved control. Yellowing, bronzing, speckling or leaf burning may occur under certain conditions. This } \\
\text { injury is generally outgrown without delaying podset or maturity. Basagran is a contact herbicide and controls many } \\
\text { young broadleaf weeds. It does not control grass. Read and follow the label directions for hard to control weeds such } \\
\text { as yellow nutsedge. }\end{array}$} \\
\hline $\begin{array}{l}\text { Carfentrazone } \\
\text { (Aim) }\end{array}$ & $\begin{array}{l}\text { Legume Group } \\
\text { (All) }\end{array}$ & $\begin{array}{l}\text { Preplant } \\
\text { Directed-hooded } \\
\text { Row-middles }\end{array}$ & 0.031 & 0.031 \\
\hline
\end{tabular}

Remarks: Aim may be applied as a preplant burndown treatment and/or as a post-directed hooded application to row middles for the burn-down of emerged broadleaf weeds. May be tank mixed with other registered herbicides. May be applied at up to $2 \mathrm{oz}$ ( $0.031 \mathrm{lb}$ ai). Use a quality spray adjuvant such as crop oil concentrate (coc) or non-ionic surfactant at recommended rates.

\begin{tabular}{|l|l|l|l|l|}
\hline $\begin{array}{l}\text { EPTC } \\
\text { (Eptam 10G) (Eptam 7E) }\end{array}$ & Beans (Green or Dry) & $\begin{array}{l}\text { Preplant incorporate } \\
\text { or at layby }\end{array}$ & $3.0-4.0$ & --- \\
\hline
\end{tabular}

Remarks: Controls germinating annuals and suppresses nutsedge and other perennial weeds. Incorporate in same operation to reduce evaporation loss. Direct layby applications between rows and incorporate.

\begin{tabular}{l|l|l}
\hline $\begin{array}{l}\text { Glyphosate } \\
\text { (Roundup, Durango, } \\
\text { Touchdown, Glyphomax) }\end{array}$ & Beans (all); Peas (all) & $\begin{array}{l}\text { Chemical fallow } \\
\text { Preplant, pre } \\
\text { emergence, } \\
\text { Pretransplant }\end{array}$ \\
\hline
\end{tabular}

Remarks: Roundup, Glyphomax and Touchdown have several formulations. Check the label of each for specific labeling directions.

\begin{tabular}{|l|l|l|l|l|}
\hline $\begin{array}{l}\text { Halosulfuron } \\
\text { (Sandea) }\end{array}$ & Snap Beans & Preemergence & $0.024-0.032$ & $0.024-0.032$ \\
\hline
\end{tabular}

Remarks: Apply preemergence application after planting but before cracking at $1 / 2$ to $2 / 3$ oz product/A. Use the lower rate on light sandy soils. Do not incorporate. For post emergence applications apply $1 / 2$ to $2 / 3$ oz product 3 weeks after emergence or at the 3 trifoliate stage, but before flowering. Do not exceed 1 oz per acre per crop cycle. Apply with a non-ionic surfactant or crop oil concentrate.

\begin{tabular}{|l|l|l|c|c|}
\hline Imazethapyr (Pursuit) & $\begin{array}{l}\text { Dry Beans, Lima } \\
\text { Beans, Southern } \\
\text { Peas, English Peas }\end{array}$ & $\begin{array}{l}\text { Preplant } \\
\text { incorporated; } \\
\text { Preemergence; } \\
\text { Early Postemergence }\end{array}$ & $0.031-0.062$ & ---- \\
\hline
\end{tabular}

Remarks: May be applied to Navy, Great Northern, Red Kidney, Black turtle, Cranberry and small white type dry beans, Lima Beans, Southern and English Peas. May be applied preplant incorporated or Preemergence to all the above crops at 2 ozs/acre or 3 to 4 ozs/acre for English and Southern Peas. An early postmergence application at 3 ozs. (English Peas) and 4 ozs./acre (Southern Peas) may be made with a non-ionic surfactant. Controls a large number of broadleaf weeds preemergence and several postemergence. Read the label for weed species and timing for control.

\begin{tabular}{|l|l|l|l|l|}
\hline $\begin{array}{l}\text { Imazethapyr } \\
\text { (Pursuit) }\end{array}$ & Snap beans & $\begin{array}{l}\text { Preplant incorporated } \\
\text { Preemergence }\end{array}$ & 0.023 & 0.023 \\
\hline
\end{tabular}

Remarks: May be applied as a preplant incorporated or preemergence treatment to snapbeans at $1.5 \mathrm{oz}$. product/acre. May be tank-mixed with a registered preemergent grass herbicide. There is a 30 day PHI. Check plant back restrictions on the label. 
Table 1. Chemical weed controls: Beans and Peas.

\begin{tabular}{|c|c|c|c|c|}
\hline \multirow[t]{2}{*}{ Herbicide } & \multirow[t]{2}{*}{ Labeled crops } & \multirow{2}{*}{$\begin{array}{c}\text { Time of } \\
\text { application to crop }\end{array}$} & \multicolumn{2}{|c|}{ Rate (Ibs. Al./Acre) } \\
\hline & & & Mineral & Muck \\
\hline $\begin{array}{l}\text { S-Metolachlor (Dual } \\
\text { Magnum) }\end{array}$ & $\begin{array}{l}\text { Pod Crops: Bush, } \\
\text { Pole, Lima, Mung } \\
\text { Beans; Southern, } \\
\text { English Peas }\end{array}$ & $\begin{array}{l}\text { Preplant incorporate } \\
\text { or preemergence }\end{array}$ & $4.5-1.26$ & 1.26 \\
\hline \multicolumn{5}{|c|}{$\begin{array}{l}\text { Remarks: Dual Magnum is an isomer of Dual and has a lower application rate. Use } 1 \text { to } 1.33 \text { pints/A. Controls most } \\
\text { annual grasses and some broadleaf weeds as well as yellow nutsedge. May be applied preplant incorporated or } \\
\text { preemergence and watered into the soil. See label for specific tank-mix combinations and recommendations for } \\
\text { Eptam and Treflan. }\end{array}$} \\
\hline $\begin{array}{l}\text { Paraquat } \\
\text { (Firestorm) } \\
\text { (Gramoxone Inteon) }\end{array}$ & $\begin{array}{l}\text { Lima, Snap Beans; } \\
\text { Peas }\end{array}$ & $\begin{array}{l}\text { Preplant } \\
\text { Preemergence }\end{array}$ & $\begin{array}{l}0.47-0.94 \\
0.31-0.47\end{array}$ & $\begin{array}{c}0.47-0.94 \\
0.5-1.0\end{array}$ \\
\hline \multicolumn{5}{|c|}{$\begin{array}{l}\text { Remarks: Apply as a band treatment over the crop row or as a broadcast treatment before, during or after planting, } \\
\text { but before the emergence of the crop. Weeds emerging after the application will not be controlled. Crop plants } \\
\text { emerged at the time of application will be killed. Use a non-ionic surfactant in the spray mixture. }\end{array}$} \\
\hline $\begin{array}{l}\text { Paraquat } \\
\text { (Firestorm) }\end{array}$ & Dry beans & Harvest aid & $0.31-0.5$ & $0.31-0.5$ \\
\hline \multicolumn{5}{|c|}{$\begin{array}{l}\text { Remarks: Use a non-ionic spreader at } 1 \text { qt. per } 100 \text { gals. of spray mix. May be used in up to } 2 \text { applications. A split } \\
\text { application may improve vine coverage. Do not harvest within } 7 \text { days of last application. May be used on the dry forms } \\
\text { of the following: Chick peas, Garbanzo beans; Sweet, White sweet, White and Grain lupines; Kidney, Lima, Mung, } \\
\text { Navy, Pinto, Snap and Wax beans; Asparagus beans; Blackeye and Cowpeas. Do not use on Faba beans. }\end{array}$} \\
\hline Pedimethalin (Prowl) & $\begin{array}{l}\text { Beans: Dry, Lima, } \\
\text { Snap; Chickpeas, } \\
\text { Southern Peas }\end{array}$ & Preplant incorporated & $0.5-0.75$ & 1.0 \\
\hline \multicolumn{5}{|c|}{$\begin{array}{l}\text { Remarks: Incorporate within } 7 \text { days of application to the top } 1 \text { to } 2 \text { inches of soil. Label state control of many weeds } \\
\text { including crabgrass, fall and Texas panicum, goosegrass, signalgrass, carpetweed, Florida pusley, kochia, } \\
\text { lambsquarter, pigweed, purslane and annual spurges. May be applied alone or tank-mixed with Dual or Eptam. }\end{array}$} \\
\hline Pelargonic Acid (Scythe) & $\begin{array}{l}\text { Legume vegetables } \\
\text { (Beans (all) Peas } \\
\text { (all)) }\end{array}$ & $\begin{array}{l}\text { Vegetative Burndown } \\
\text { (site preparation }\end{array}$ & $3-10 \% \mathrm{v} / \mathrm{v}$ & $3-10 \% \mathrm{v} / \mathrm{v}$ \\
\hline \multicolumn{5}{|c|}{$\begin{array}{l}\text { Remarks: General contact, non-selective, foliar applied herbicide. No residual control. Product is non-translocated. } \\
\text { May be tank mixed with soil residual compounds. Consult label for rates and other information. }\end{array}$} \\
\hline $\begin{array}{l}\text { Quizalofop (Assure II) } \\
\text { (Targa) }\end{array}$ & $\begin{array}{l}\text { Snap beans, dry } \\
\text { beans, succulent and } \\
\text { dry peas }\end{array}$ & Postemergence & $0.04-0.08$ & $0.04-0.08$ \\
\hline \multicolumn{5}{|c|}{$\begin{array}{l}\text { Remarks: For control of emerged annual and perennial grasses. Application is } 6-12 \text { oz actively growing grasses } \\
\text { depending on species to be controlled. Subsequent flushes of grasses require additional treatments. For ground } \\
\text { application always include a nonphytotoxic petrolium based oil concentrate at } 1 \% \mathrm{v} / \mathrm{v}(4 \mathrm{qts} / 100 \mathrm{gals}) \text { or a non ionic } \\
\text { surfactant at } 0.25 \% \mathrm{v} / \mathrm{v}(1 \mathrm{qt} / 100 \mathrm{gal}) \text {. Reductions in grass control is possible when applied immediately prior to, or } \\
\text { sequentially after application of post broadleaf herbicides. Follow label directions. It may be tank mixed with } \\
\text { Basagran. Do not apply within } 15 \text { days of harvest. Do not apply more than } 14 \mathrm{oz} \text { of product per acre per season. }\end{array}$} \\
\hline Sethoxydim (Poast) & $\begin{array}{l}\text { Beans and Peas, dry } \\
\text { and succulent }\end{array}$ & Postemergence & $0.188-0.28$ & ---- \\
\hline
\end{tabular}


Table 1. Chemical weed controls: Beans and Peas.

\begin{tabular}{|c|c|c|c|c|}
\hline \multirow[t]{2}{*}{ Herbicide } & \multirow[t]{2}{*}{ Labeled crops } & \multirow{2}{*}{$\begin{array}{c}\text { Time of } \\
\text { application to crop }\end{array}$} & \multicolumn{2}{|c|}{ Rate (Ibs. Al./Acre) } \\
\hline & & & Mineral & Muck \\
\hline \multicolumn{5}{|c|}{$\begin{array}{l}\text { Remarks: For postemergence control of annual and perennial grass weeds, use } 1 \text { pt. ( } 0.188 \mathrm{lb} \text {. ai.) to } 1.5 \text { pts. (0.28 } \\
\text { Ib. ai.) per acre depending on weed species to be controlled. Will not control sedges or broadleaf weeds. Use } 2 \text { pts. } \\
\text { crop oil concentrate per acre in the spray mix. Do not apply more then } 4 \text { pts. per acre in one season. Do not apply } \\
\text { within } 15 \text { days of harvest for succulent beans and peas or } 30 \text { days for dry beans and peas. Bean and pea types and } \\
\text { species on which application may be made include beans of the Phaseolus genus (includes Adzuki bean, Field bean, } \\
\text { Kidney bean, Lima bean, Navy bean, Mung bean); Lupines (includes Sweet lupine, White lupine, Grain lupine); } \\
\text { Cowpeas - Vigna species (includes blackeye pea, Southern pea, Broad bean); Vicia faba or faba bean; Chick pea - } \\
\text { Cicer arietinum or garbanzo bean; guar - Cyamopsis tetragoneloba; and peas - Pisum species (includes garden peas, } \\
\text { field peas, sugar peas). }\end{array}$} \\
\hline Sodium Chlorate (Defol 6) & $\begin{array}{l}\text { Dry beans; Southern } \\
\text { peas; Guar beans }\end{array}$ & Defoliant/Desiccant & 6.0 & 6.0 \\
\hline \multicolumn{5}{|c|}{$\begin{array}{l}\text { Remarks: Apply at a rate of } 1 \text { gal. per acre in } 5 \text { to } 10 \text { gals. of water by air or } 10 \text { to } 20 \text { gal. by ground equipment. } \\
\text { Thorough coverage is essential. Make application } 7 \text { to } 10 \text { days before anticipated harvest, longer if temperatures are } \\
\text { cool. Do not graze treated fields or feed treated fodder or forage to livestock. }\end{array}$} \\
\hline $\begin{array}{l}\text { Trifluralin } \\
\text { (Treflan EC) } \\
\text { (Treflan TR-10) } \\
\text { (Trilin) }\end{array}$ & $\begin{array}{l}\text { Green, Lima, Mung, } \\
\text { Guar Beans; } \\
\text { Southern, English } \\
\text { Peas }\end{array}$ & Preplant incorporated & $0.5-0.75$ & ---- \\
\hline
\end{tabular}

